\title{
The Ground Clutter Canceller based on the Clutter Recovery in Airborne MIMO Radar
}

\author{
Jian ZHOU, Yan ZHOU*, Lin WANG, Bo JIANG, Cai WEN \\ School of Information Science and Technology \\ Northwest University \\ Xi'an 710127, China \\ *E-mail: yanzhou@nwu.edu.cn \\ *Corresponding author
}

\begin{abstract}
Current researches show that the clutter rank is much lower than the system degrees of freedom (DoFs) in the airborne MIMO radar with orthogonal waveforms. According to this conclusion, we propose the ground clutter canceller based on the clutter recovery (CCCR) to suppress the clutter and reduce the DoFs of the clutter. Several linearly independent space-time steering-vectors are constructed to recover the original clutter received by the MIMO radar. Then clutter echoes received in adjacent pulses are maximally cancelled to build our clutter canceller. The clutter canceller can be cascaded with the conventional spatial-temporal matching or dimension-reduced STAP to form the two-stage pre-filter and detector. As the pre-filter, the CCCR can evidently reduce the clutter DoFs, leaving more system DoFs available for detecting moving targets. Therefore, the moving target detection performance of the following spatial-temporal matching or dimension-reduced STAP will definitely outperform the original one.
\end{abstract}

Keywords-multiple-input multiple-output (MIMO) radar; space-time adaptive processing (STAP); clutter suppression

\section{INTRODUCTION}

Since the concept of multiple-input multiple-output (MIMO) radar first proposed in [1], it has received considerable attention for its superiority in spatial diversity and available degrees of freedom (DoFs) compared with the traditional single-input multiple-output (SIMO) radar [1-3]. More recently, the researches on MIMO radar have been extended to the space-time adaptive processing (STAP) [4-6], which is an important technique in the airborne radar due to its remarkable ability of the clutter suppression and the moving target detection. The STAP in MIMO radar will obtain better target detection performance for more available system DoFs. However, the MIMO STAP will face more severe clutter environment because of the extra dimension introduced by transmitting orthogonal waveforms.

Clutter rank, which is characterized by the number of dominant eigenvalues of the clutter covariance, is a key point in STAP since it implies how many remaining system DoFs can be exploited to detect moving target. According to the conclusion in [3], in MIMO radar with orthogonal waveforms, the clutter rank is rather lower than the system DoFs. In other words, the ground clutter can be recovered by a small number of linearly independent vectors in clutter space [8]. Hence, inspired by this idea, we propose the ground clutter canceller based on the clutter recovery (CCCR) to further enhance the clutter suppression and the moving target detection ability in the airborne MIMO radar.

\section{THE GRound ClutTER CANCELLER BASED ON THE Clutter Recovery}

The airborne MIMO radar system to be studied is moving at a constant speed $v_{a}$ parallel to the ground. The transmitting array consists of $M$ array elements with interelement space ${ }^{d_{t}}$ and the receiving array consists of $N$ array elements with interelement space $d_{r}$. The MIMO radar simultaneously transmits $M$ orthogonal waveforms. The $l$ th range cell is uniformly divided into $N_{c}$ independent clutter patches without consideration of the earth curvature. The location of the $i$ th clutter patch is described by its azimuth angle $\theta_{i}$ and elevation angle $\varphi_{l}$. Given these parameters, the normalized Doppler frequency can be expressed as $f_{d i}=2 T_{r} v_{a} \cos \theta_{i} \cos \varphi_{l} / \lambda$ Assuming that the MIMO radar system transmits $K$ pulses on the repetition of $T_{r}$ in one CPI, the clutter data received during a CPI can be arranged in the vector form as [3]

$$
\mathbf{y}(l)=\sum_{i=1}^{N_{c}} \beta_{i} \boldsymbol{s}_{t}\left(f_{d i}\right) \otimes \boldsymbol{s}_{s}\left(\theta_{i}, \varphi_{l}\right)=\sum_{i=1}^{N_{c}} \beta_{i} \mathbf{c}_{i},
$$

where $\mathbf{c}_{i}=\boldsymbol{s}_{t}\left(f_{d i}\right) \otimes \boldsymbol{s}_{s}\left(\theta_{i}, \varphi_{l}\right)$ is the space-time steering-vector

$\boldsymbol{s}_{t}\left(f_{d i}\right)=\left[1, \exp \left(j 2 \pi f_{d i}\right), \cdots, \exp \left(j 2 \pi(K-1) f_{d i}\right)\right]^{T}$ with $\boldsymbol{s}_{s}\left(\theta_{i}, \varphi_{l}\right)=\boldsymbol{a}_{t}\left(\theta_{i}, \varphi_{l}\right) \otimes \boldsymbol{a}_{r}\left(\theta_{i}, \varphi_{l}\right) \quad$ representing the temporal and spatial steering-vector, respectively, $\bigotimes$ is the Kronecker product. As stated in [8], the dimension of the clutter subspace that spanned by $\left[\mathbf{c}_{1}, \mathbf{c}_{2}, \cdots, \mathbf{c}_{N_{c}}\right]$ is $r_{c}=\lfloor N+\alpha(M-1)+\beta(K-1)\rfloor$, where $\alpha=d_{t} / d_{r}$, the brackets \lfloor\rfloor indicate rounding to the nearest integer. Therefore, the clutter received by the airborne MIMO radar can be recovered by $r_{c}$ linearly independent space-time steering-vectors in the clutter subspace, namely 


$$
\mathbf{y}(l)=\sum_{r=1}^{r_{c}} \alpha_{r} \mathbf{g}_{r}=\boldsymbol{\Psi} \boldsymbol{\gamma}
$$

$$
\text { where } \quad \boldsymbol{\Psi}=\left[\mathbf{g}_{1}, \cdots, \mathbf{g}_{r}, \cdots, \mathbf{g}_{r_{c}}\right]
$$

$\boldsymbol{\gamma}=\left[\alpha_{1}, \cdots, \alpha_{r}, \cdots \alpha_{r_{c}}\right]^{T}$ respectively contain the linearly independent space-time steering-vectors and the corresponding complex coefficients, in which

$$
\begin{aligned}
& \mathbf{g}_{r}=\left[1, \cdots, \exp \left(j 2 \pi(n-1+\alpha(m-1)+\beta(k-1)) f_{r}\right) \cdots,\right. \\
& \left.\exp \left(j 2 \pi(N-1+\alpha(M-1)+\beta(K-1)) f_{r}\right)\right]^{T}
\end{aligned}
$$

The parameter $f_{r}=r / r_{c}, r=0,1, \cdots, r_{c}-1 \quad$ [16]. Obviously, the clutter space can be spanned by the columns of $\boldsymbol{\Psi}$.

As similar to (1), (2) can be regarded as the clutter space-time snapshots from some clutter patches. Then $\mathbf{x}(k)$ that represents clutter echoes received at the $k$ th transmitting pulse can be written as

$$
\mathbf{x}(k)=\sum_{r=1}^{r_{c}} \alpha_{r} \mathbf{g}_{r}(k),
$$

where

$\mathbf{g}_{r}(k)=\left[\exp \left(j 2 \pi(k-1) \beta f_{r}\right), \cdots, \exp \left(j 2 \pi(n-1+\alpha(m-1)+\beta(k-1)) f_{r}\right), \cdots\right.$, $\left.\exp \left(j 2 \pi(N-1+\alpha(M-1)+\beta(k-1)) f_{r}\right)\right]$

One re-express (4) as the matrix-vector form

$$
\mathbf{x}(k)=\mathbf{D F}(k) \mathbf{a},
$$

where

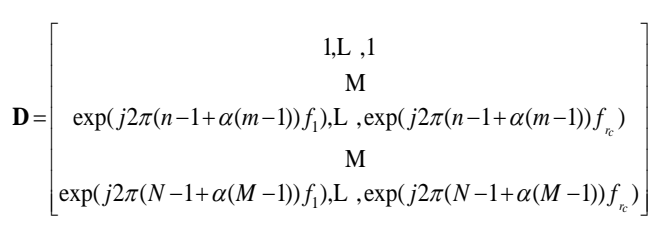

$\mathbf{F}(k)=\operatorname{diag}\left(\left[\exp \left(\mathrm{j} 2 \pi \beta(k-1) f_{1}, \mathrm{~L}, \exp \left(\mathrm{j} 2 \pi \beta(k-1) f_{r_{c}}\right]\right), \quad \mathbf{a}=\left[a_{1}, \mathrm{~L}, a_{r_{c}}\right]^{T}\right.\right.$

In order to design the CCCR, a filtering coefficient matrix $\mathbf{A}$ is established to minimize the residual clutter energy between adjacent pulses, that is

$$
\begin{aligned}
\min _{A}\|\boldsymbol{\varepsilon}(k)\|_{F}^{2} & =\min _{A}\|\mathbf{A x}(k)-\mathbf{x}(k+1)\|_{F}^{2} \\
& =\min _{A}\|\mathbf{A D F}(k) \mathbf{a}-\mathbf{D F}(k+1) \mathbf{a}\|_{F}^{2}
\end{aligned}
$$

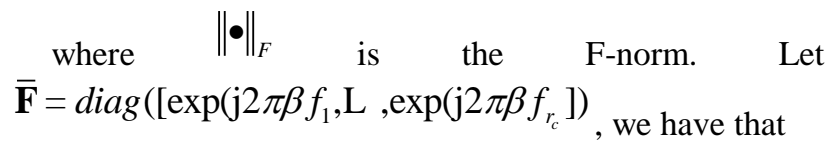

$$
\min _{A}\|\boldsymbol{\varepsilon}(k)\|_{F}^{2}=\min _{A}\left\|\mathbf{A D} \overline{\mathbf{F}}^{k-1} \mathbf{a}-\mathbf{D} \overline{\mathbf{F}} \overline{\mathbf{F}}^{k-1} \mathbf{a}\right\|_{F}^{2}=\min _{A}\left\|(\mathbf{A D}-\mathbf{D} \overline{\mathbf{F}}) \overline{\mathbf{F}}^{k-1} \mathbf{a}\right\|_{F}^{2}
$$

The solution to (9) is

$$
\mathbf{A}=\mathbf{D} \overline{\mathbf{F}} \mathbf{D}\left(\mathbf{D} \mathbf{D}^{H}\right)^{-1} .
$$

The filtering coefficient matrix $\mathbf{A}$ can be computed if the matrices $\mathbf{D}$ and $\overline{\mathbf{F}}$ are known. The matrices $\mathbf{D}$ and $\overline{\mathbf{F}}$ are only determined by the parameters defined in (7). Thus, if the clutter rank and radar operating parameters are known in advance, the matrices $\mathbf{D}$ and $\overline{\mathbf{F}}$ can be utilized as the prior information for computing $\mathbf{A}$. In other words, the CCCR filtering coefficient matrix $\mathbf{A}$ can be pre-calculated off-line

\section{The Proposed Two-STAGE ClutTer FILTER}

In fact, the CCCR proposed can be exploited as a clutter pre-filter before the spatial-temporal matching or dimension-reduced STAP to form the two-stage clutter filter. In the first stage, the CCCR as the pre-filter can effectively filter out most of the clutter. The second stage, which is often the spatial-temporal matching or dimension-reduced STAP, can further suppress the residual clutter and detect the moving target.

The space-time data received by the MIMO radar system in a CPI rearranged into a vector can be expressed as

$$
\mathbf{x}=\left[x_{1,1,1}, x_{1,1,2}, \mathrm{~L}, x_{k, m, n} \mathrm{~L}, x_{K, M, N}\right] \text {. }
$$

Assuming that the spatial-temporal steering-vector of the target is expressed as $\mathbf{S}=\mathbf{s}_{t} \otimes \mathbf{S}_{s}$, where $\mathbf{s}_{t} \in \mathbf{C}^{K \times 1}$ and $\mathbf{s}_{s} \in \mathbf{C}^{M N \times 1}$, respectively, are the temporal and spatial target steering-vectors. A pre-filtering matrix of the CCCR is devised as

$$
\mathbf{H}=\left[\begin{array}{cccccc}
\mathbf{D} & -\mathbf{I}_{M N} & & & & \\
& \mathbf{D} & -\mathbf{I}_{M N} & & \mathbf{0} & \\
& & \mathrm{O} & \mathrm{O} & & \\
& \mathbf{0} & & \mathbf{D} & -\mathbf{I}_{M N} & \\
& & & & \mathbf{D} & -\mathbf{I}_{M N}
\end{array}\right] \in \mathbf{C}^{(K-1) M N \times K M N}
$$

where $\mathbf{I}_{M N}$ is an $M N \times M N$ identity matrix. The CCCR pre-filter as the first stage is suppressing the ground clutter, namely

$$
\overline{\mathbf{x}}=\mathbf{H} \mathbf{x}
$$

The following moving target detection methods as the second stage are illustrated separately: 

$\mathbf{S}$ is

1) Spatial-temporal matching

The CCCR pre-filtering operation on the target vector

$$
\overline{\mathbf{s}}=\mathbf{H s} .
$$

Correspondingly, the weight vector of the spatial-temporal matching is

$$
\mathbf{w}_{s t}=\mathbf{H}\left(\left(\mathbf{s}_{t} \bullet \mathbf{b}_{t}\right) \otimes\left(\mathbf{s}_{s} \bullet \mathbf{b}_{s}\right)\right),
$$

where - is the Hadamard product implying the element-by-element operation of the two vectors, $\mathbf{b}_{t} \in \mathbf{C}^{K \times 1}$ and $\mathbf{b}_{s} \in \mathbf{C}^{M N \times 1}$ are the temporal and spatial static window vectors adopted for attenuating the sidelobes, respectively, such as the well-known Chebyshev window, Hamming window and so on.

Dimension-reduced STAP

Supposed that the corresponding dimension-reduced matrix for the STAP is $\mathbf{T} \in \mathbf{C}^{r \times(K-1) M N}$, then a dimension-reduced operation is made on the pre-filtered data and target steering-vector

$$
\mathbf{H}=\mathbf{T} \overline{\mathbf{x}}
$$

$$
\S 0 \mathbf{T} \overline{\mathbf{s}}
$$

As a result, the dimension-reduced space-time filter is:

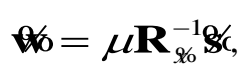

where $\mu$ is a constant and $\mathbf{R}_{\text {Yoo }}=E\left[\mathbf{8} \mathbf{H}^{H}\right]$ is the correlation matrix of the dimension-reduced data ${ }_{c}$. It is often taken place of by the maximum likelihood estimation of itself.

\section{Simulation RESUlts}

In this experiments, the orthogonal quadrature phase shift keyed (QPSK) signal with snapshots $P=128$ is transmitted by the airborne MIMO radar where both the uniform linear transmitting array and receiving array are $M=N=5$ antennas. The interelements of the transmitting array and receiving array are $d_{t}=0.2 \mathrm{~m}$ and $d_{r}=0.1 \mathrm{~m}$, respectively. In a CPI, $K=16$ pulses are transmitting at a pulse repetition frequency $f_{r}=2000 \mathrm{~Hz}$.
The platform is $h_{a}=9 \mathrm{Km}$ high at a speed of $v_{a}=100 \mathrm{~m} / \mathrm{s}$. The CNR (clutter to noise ratio) is $60 \mathrm{~dB}$. An SNR (signal to noise ratio) OdB moving target is injected into the under-detection range cell with Doppler frequency $f_{d r}=0.2 f_{r}$.

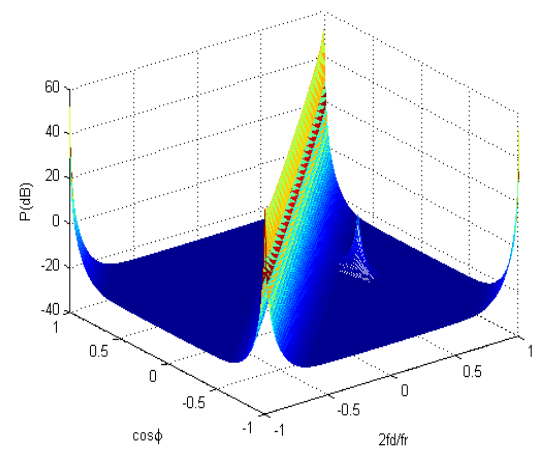

(a) The original clutter spectral

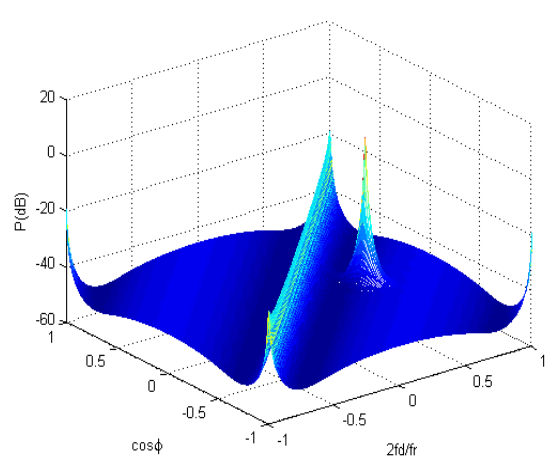

(b) The clutter spectral after pre-filtering

Figure 1. The clutter spectral before and after the pre-filtering

Fig.1(a) shows the original clutter spectral. The clutter ridge is distributed along the diagonal in the angle-Doppler plane. Compared with the strong clutter, the target which is located on the right side of the clutter ridge is much weaker. Fig. 1(b) shows that after filtering, the power of the clutter is sharply reduced. Obviously, the power of the moving target is considerably stronger than the main clutter. This indicates that the CCCR can effectively suppress the clutter by forming the nulling along the clutter ridge. It is worth noting that the CCCR pre-filter facilities the following detection of the moving target. 


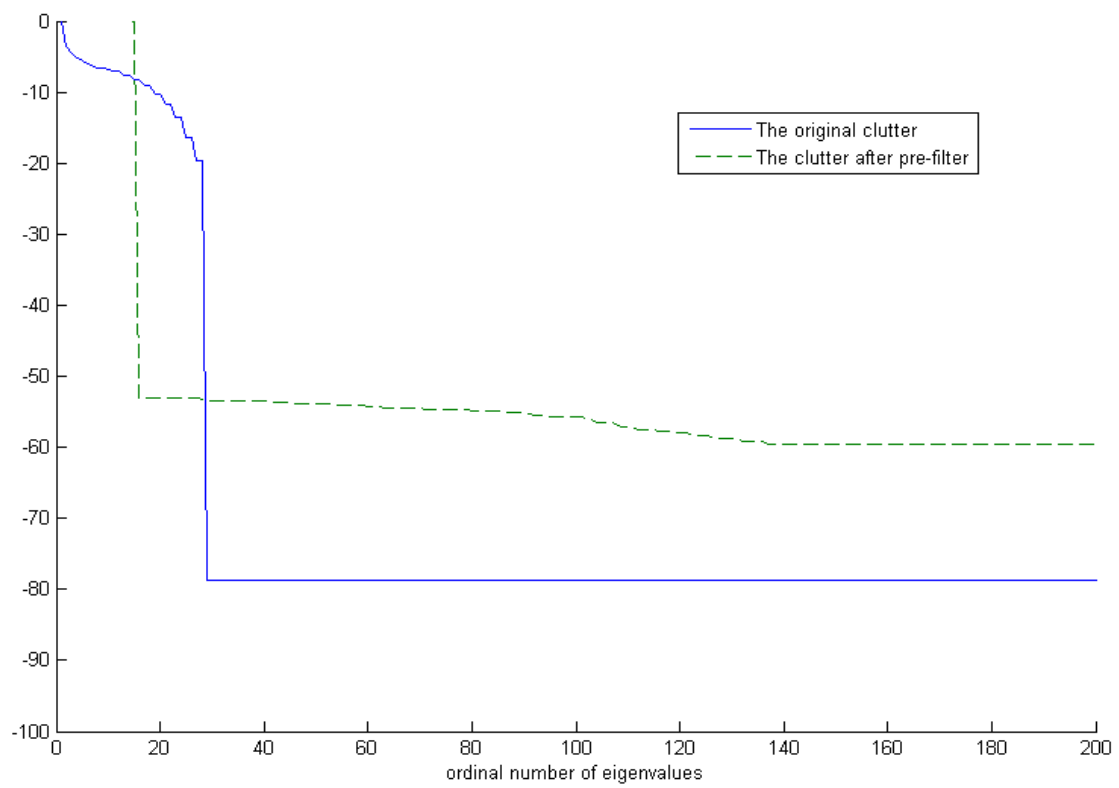

Figure 2. The eigenvalue distribution of the clutter covariance

Fig. 2 shows the eigenvalue distribution of the clutter covariance before and after the CCCR pre-filtering. In general, the clutter rank is characterized by the number of the dominant eigenvalues. According to the setting MIMO radar parameters, the theoretical number of the dominant eigenvalues is about 28 , which is totally satisfied with the experimental result shown in Fig. 3. The dotted line shows that after CCCR pre-filtering, the number of the dominant eigenvalues is apparently reduced to less than 20. Low clutter DoFs implies that more system DoFs are available for moving target detection

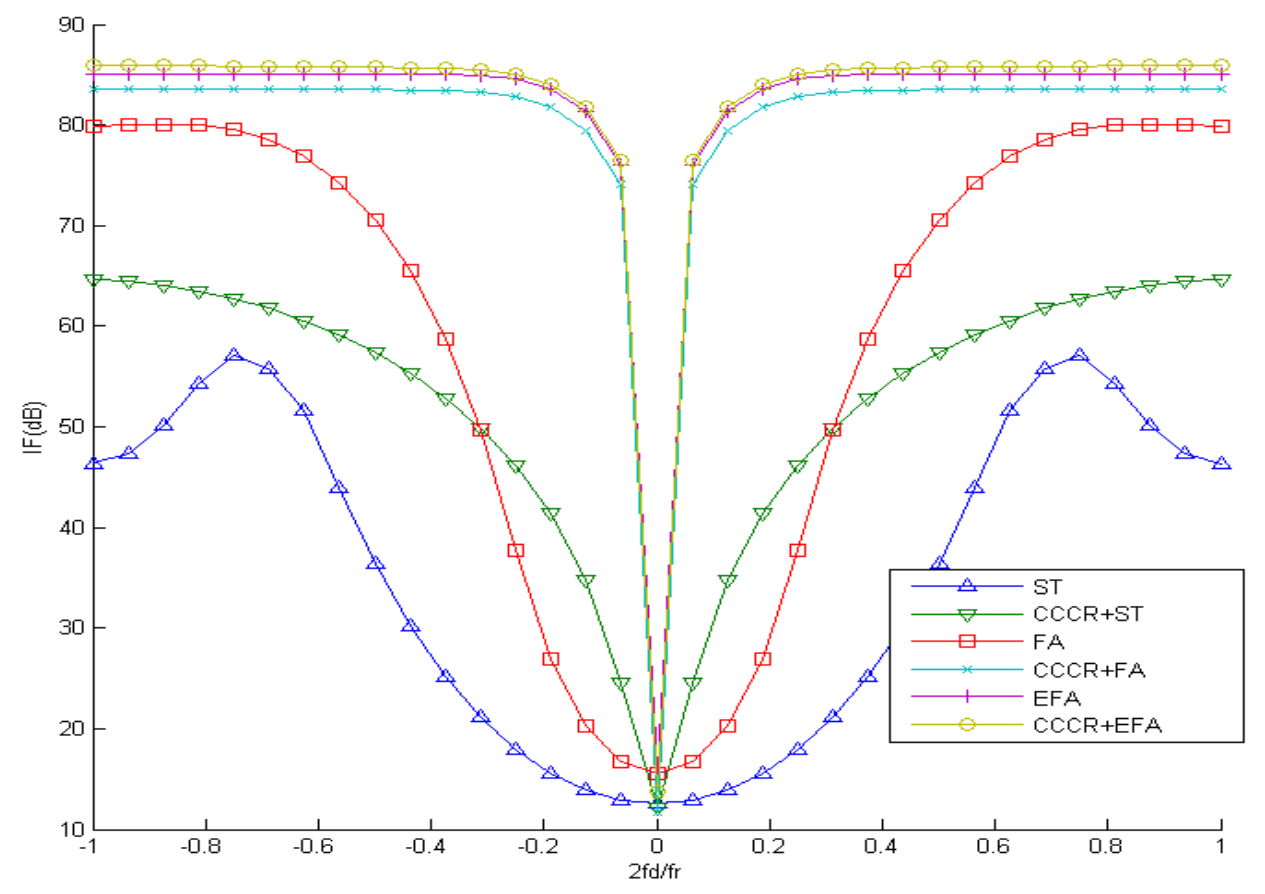

Figure 3. The IF performance before and after the pre-filter

Improvement factor (IF) defined as the ratio of the output SINR (signal to interference and noise ratio) and input SINR is an important scale to measure the detection performance of the STAP algorithm. In this section, ST 
means spatial-temporal matching. $\mathrm{CCCR}+\mathrm{ST}, \mathrm{CCCR}+\mathrm{FA}$ and CCCR+EFA represent the original spatial-temporal matching, FA and EFA cascaded with the CCCR pre-filter, respectively. It is presented in Fig.3 that the IF performances of ST, FA and EFA are improved after cascaded with the CCCR pre-filter. Near the main clutter region at the normalized Doppler frequency $f_{d} / f_{r}=0.1$, the IF curves of the CCCR+ST CCCR+FA and CCCR+EFA are, respectively, better than the original ST, FA and EFA by about $30 d B, 60 d B$ and $0.8 d B$. Away from the main clutter region at normalized Doppler frequency $f_{d} / f_{r}=0.3$, the IF curves of them are, respectively, better than the original ST, FA and EFA by about $12 d B, 7 d B$ and $1.2 d B$. The obvious IF performance improvement in main clutter region for the STAP algorithm benefits the slow moving target detection.

\section{CONCLUSIONS}

In this paper, a CCCR pre-filter that can effectively suppress the ground clutter in the airborne MIMO radar is proposed. The clutter is recovered by $r_{c}$ linearly independent steering-vectors in clutter space and then adjacent clutter echoes are cancelled to a maximally extent. Since it can decrease the DoFs of the clutter, the performance of the conventional spatial-temporal matching or dimension-reduced STAP can be enhanced after the CCCR pre-filter.

\section{ACKNOWLEDGEMENT}

This research was financially supported by the National Science Foundation of China under Grant 61373177, 61503300 and 41601353.

\section{REFERENCES}

[1] D. W. Bliss and K. W. Forsythe, Multiple-input multiple-output (MIMO) radar and

[2] imaging: Degrees of freedom and resolution, in Proc. 37th IEEE Asilomar Conf. Signal, Systems, Computers, vol. 1, pp. 54-49, 2003.

[3] E. Fisher, A. Haimovich, and R. Blum, et al, Spatial diversity in radars-Models and

[4] detection performance, IEEE Trans. Signal Process., vol. 20, pp. 823-838, 2006.

[5] C. Y. Chen and P. P. Vaidyanathan, MIMO radar space-time adaptive processing

[6] using prolate spheroidal wave functions, IEEE Trans. Signal. Process., vol. 56, no. 2, pp.623-635, 2007.

[7] Y Li, S A Vorobyovz, Z He, Joint hot and cold clutter mitigation in the transmit

[8] beamspace-based MIMO radar, IEEE International Conference on Acoustics, Speech and Signal Processing, 2015.

[9] A F Liu, H B Sun, K C Ten, etc, Robust space-time adaptive processing for

[10] nonhomogeneous clutter in the presence of model errors, IEEE Trans. Aerosp. Electron. Syst., vol. 52, no. 1, pp. 155-168, 2016.

[11] J. Ward, Space-time adaptive processing for airborne radar, MIT Lincoln Lab.,

[12] Lexington, MA, Tech. Rep. 1015, 1994.

[13] N. A. Goodman and J. M.Stiles, On clutter rank observed by arbitrary arrays, IEEE
[14] Trans. Signal Process., vol. 55, pp. 168-178, 2007.

[15] Z. C. Yang, X. Li, and H. Q. W, et al, On clutter sparsity analysis in space-time

[16] adaptive processing airborne radar, IEEE Signal. Process. Lett., vol. 10, no. 5, pp. 1214-1217, 2013.

[17] R. C. Dippetro, Extended factored space-time processing for airborne radar system,

[18] in Proc. 26th Asilomar Conf. Signals, Systems and Computers, October 1992, pp. 425-430.

[19] Z. Bao, G. S. Liao and B. R. Wu, et al, Adaptive spatial-temporal processing for

[20] airborne radars, Chin. J. Electron, vol.2, no.1, pp. 2-7, 1993. 